\title{
STIELTJES POLYNOMIALS AND RELATED QUADRATURE FORMULAE FOR A CLASS OF WEIGHT FUNCTIONS
}

\author{
WALTER GAUTSCHI AND SOTIRIOS E. NOTARIS
}

\begin{abstract}
Consider a (nonnegative) measure $d \sigma$ with support in the interval $[a, b]$ such that the respective orthogonal polynomials, above a specific index $\ell$, satisfy a three-term recurrence relation with constant coefficients. We show that the corresponding Stieltjes polynomials, above the index $2 \ell-1$, have a very simple and useful representation in terms of the orthogonal polynomials. As a result of this, the Gauss-Kronrod quadrature formulae for $d \sigma$ have all the desirable properties, namely, the interlacing of nodes, their inclusion in the closed interval $[a, b]$ (under an additional assumption on $d \sigma$ ), and the positivity of all weights. Furthermore, the interpolatory quadrature formulae based on the zeros of the Stieltjes polynomials have positive weights, and both of these quadrature formulae have elevated degrees of exactness.
\end{abstract}

\section{INTRODUCTION}

Consider a (nonnegative) measure $d \sigma$ with support in the interval $[a, b]$, and let $\pi_{n}(\cdot)=\pi_{n}(\cdot ; d \sigma)$ be the respective monic orthogonal polynomial of degree $n$. The corresponding monic Stieltjes polynomial $\pi_{n+1}^{*}(\cdot)=\pi_{n+1}^{*}(\cdot ; d \sigma)$, of degree $n+1$, can be uniquely defined by the orthogonality condition

$$
\int_{a}^{b} \pi_{n+1}^{*}(t) t^{k} \pi_{n}(t) d \sigma(t)=0, \quad k=0,1, \ldots, n
$$

(see $[2, \S 4])$, that is, $\pi_{n+1}^{*}$ is orthogonal to all polynomials of lower degree relative to the variable-sign distribution $d \sigma^{*}(t)=\pi_{n}(t) d \sigma(t)$.

Related to $\pi_{n+1}^{*}$ is the Gauss-Kronrod quadrature formula for $d \sigma$,

$$
\int_{a}^{b} f(t) d \sigma(t)=\sum_{\nu=1}^{n} \sigma_{\nu} f\left(\tau_{\nu}\right)+\sum_{\mu=1}^{n+1} \sigma_{\mu}^{*} f\left(\tau_{\mu}^{*}\right)+R_{n}^{K}(f),
$$

where $\tau_{\nu}=\tau_{\nu}^{(n)}$ are the zeros of $\pi_{n}$, and the nodes $\tau_{\mu}^{*}=\tau_{\mu}^{*(n)}$ and all weights $\sigma_{\nu}=\sigma_{\nu}^{(n)}, \sigma_{\mu}^{*}=\sigma_{\mu}^{*(n)}$ are chosen such that (1.2) has maximum degree of exactness (at least) $3 n+1$, i.e., $R_{n}^{K}(f)=0$ for all $f \in \mathbb{P}_{3 n+1}$. A necessary and sufficient condition for this is that the $\tau_{\mu}^{*}$ be the zeros of $\pi_{n+1}^{*}$ (see [5, Corollary]).

Received by the editor November 15, 1994.

1991 Mathematics Subject Classification. Primary 33C45, 65D32.

Key words and phrases. Stieltjes polynomials, Gauss-Kronrod quadrature formulae, interpolatory quadrature formulae. 
Also connected with $\pi_{n+1}^{*}$ is the interpolatory quadrature formula

$$
\int_{a}^{b} f(t) d \sigma(t)=\sum_{\mu=1}^{n+1} w_{\mu}^{*} f\left(\tau_{\mu}^{*}\right)+R_{n}^{S}(f),
$$

where $\tau_{\mu}^{*}=\tau_{\mu}^{*(n)}$ are the zeros of $\pi_{n+1}^{*}$. This kind of quadrature formula was first considered by Monegato in [10, Part II.1] for the Legendre measure $d \sigma(t)=d t$ on $[-1,1]$; he conjectured, in this case, that the $w_{\mu}^{*}$ are all positive.

We now assume that the orthogonal polynomials relative to $d \sigma$ satisfy a threeterm recurrence relation of the following kind,

$$
\begin{gathered}
\pi_{n+1}(t)=\left(t-\alpha_{n}\right) \pi_{n}(t)-\beta_{n} \pi_{n-1}(t), n=0,1,2, \ldots, \\
\alpha_{n}=\alpha, \beta_{n}=\beta \text { for } n \geq \ell,
\end{gathered}
$$

where $\alpha_{n} \in \mathbb{R}, \beta_{n}>0, \ell \in \mathbb{N}$, and $\pi_{0}(t)=1, \pi_{-1}(t)=0$. Thus, the coefficients $\alpha_{n}$ and $\beta_{n}$ are constant equal, respectively, to some $\alpha \in \mathbb{R}$ and $\beta>0$ for $n \geq \ell$. Any such measure $d \sigma$ is known to be supported on a finite interval [8, Theorem 10], say $[a, b]$, and we indicate this, together with the property (1.4), by writing $d \sigma \in \mathcal{M}_{\ell}^{(\alpha, \beta)}[a, b]$. We show in $\S 2$ that, if $d \sigma \in \mathcal{M}_{\ell}^{(\alpha, \beta)}[a, b]$, then $\pi_{n+1}^{*}(\cdot ; d \sigma)$ has a very simple and convenient representation (see $(2.13))$ in terms of $\pi_{n+1}(\cdot ; d \sigma)$ and $\pi_{n-1}(\cdot ; d \sigma)$, provided that $n \geq 2 \ell-1$. Subsequently in $\S 3$, this representation is used to derive a number of properties for the Gauss-Kronrod formula (1.2), namely that the nodes $\tau_{\mu}^{*}$ interlace with the nodes $\tau_{\nu}$, all nodes $\tau_{\nu}, \tau_{\mu}^{*}$ are contained in $[a, b]$ (under an additional assumption on $d \sigma$ ), all weights $\sigma_{\nu}, \sigma_{\mu}^{*}$ are positive, and the degree of exactness is at least $4 n-2 \ell+2$. Moreover, in $\S 4$ we show that the interpolatory formula (1.3) has positive weights and degree of exactness $2 n-1$.

Among the many orthogonal polynomials satisfying (1.4) we mention the four Chebyshev-type polynomials and their modifications discussed in Allaway's thesis [1, Ch. 4], as well as those associated with the Bernstein-Szegö measures. For many of these, the Stieltjes polynomials have previously been expressed explicitly in terms of Chebyshev polynomials, and the corresponding Gauss-Kronrod formulae have been shown to possess the desirable properties mentioned above (see $[6,7,10$, $11,12])$. In addition, it has been shown in [12] that, for a class of Bernstein-Szegö measures, the weights in the interpolatory formula (1.3) are all positive.

\section{The Stieltues polynomials}

We now present, assuming $d \sigma \in \mathcal{M}_{\ell}^{(\alpha, \beta)}[a, b]$, the explicit formula for $\pi_{n+1}^{*}(\cdot ; d \sigma)$ in terms of the respective orthogonal polynomials $\pi_{m}(\cdot)=\pi_{m}(\cdot ; d \sigma)$. We begin with two preliminary lemmas, which play an important role in the subsequent development. Both make reference to the expansion of $t^{k} \pi_{n}(t)$ for $k=0,1, \ldots, n$ in terms of the $\pi_{m}$ 's, which we write in the form

$$
t^{k} \pi_{n}(t)=\sum_{i=-k}^{k} c_{i, k}^{n} \pi_{n+i}(t), k=0,1, \ldots, n ; n \geq 1 .
$$

Note that the terms $\pi_{n+i}$ with $i<-k$ are absent in (2.1) because of orthogonality of the $\pi_{m}$. 
Lemma 2.1. Consider a measure $d \sigma \in \mathcal{M}_{\ell}^{(\alpha, \beta)}[a, b]$. For a given $n \geq \ell$, the corresponding Stieltjes polynomial has the form

$$
\pi_{n+1}^{*}(t)=\pi_{n+1}(t)-\beta \pi_{n-1}(t)
$$

if and only if in (2.1) we have

$$
c_{-1, k}^{n}=\beta c_{1, k}^{n}, \quad k=1,2, \ldots, n .
$$

Proof. Sufficiency. Assume that (2.3) holds. To prove (2.2), it suffices to show, by virtue of (1.1), that

$$
\int_{a}^{b}\left[\pi_{n+1}(t)-\beta \pi_{n-1}(t)\right] t^{k} \pi_{n}(t) d \sigma(t)=0, \quad k=0,1, \ldots, n .
$$

For $k=0$, this is true by orthogonality. When $k=1,2, \ldots, n$, we obtain from (2.1), (2.3) and orthogonality

$$
\begin{gathered}
\int_{a}^{b}\left[\pi_{n+1}(t)-\beta \pi_{n-1}(t)\right] t^{k} \pi_{n}(t) d \sigma(t) \\
=\int_{a}^{b}\left[\pi_{n+1}(t)-\beta \pi_{n-1}(t)\right]\left[\cdots+c_{1, k}^{n} \pi_{n+1}(t)+c_{0, k}^{n} \pi_{n}(t)+\beta c_{1, k}^{n} \pi_{n-1}(t)+\cdots\right] d \sigma(t) \\
=c_{1, k}^{n}\left[\int_{a}^{b} \pi_{n+1}^{2}(t) d \sigma(t)-\beta^{2} \int_{a}^{b} \pi_{n-1}^{2}(t) d \sigma(t)\right] \\
=c_{1, k}^{n}\left(\left\|\pi_{n+1}\right\|^{2}-\beta^{2}\left\|\pi_{n-1}\right\|^{2}\right),
\end{gathered}
$$

where $\|\cdot\|$ is the $L_{2}$-norm. Since $n \geq \ell$, there follows from (1.4) that $\beta_{n}=\beta_{n+1}=\beta$, or equivalently,

$$
\frac{\left\|\pi_{n}\right\|^{2}}{\left\|\pi_{n-1}\right\|^{2}}=\frac{\left\|\pi_{n+1}\right\|^{2}}{\left\|\pi_{n}\right\|^{2}}=\beta
$$

(cf. [4, Eq. (5.3)]). This yields

$$
\frac{\left\|\pi_{n+1}\right\|^{2}}{\left\|\pi_{n-1}\right\|^{2}}=\beta^{2}
$$

which, inserted in the last equality of (2.5), proves $(2.4)$ for $k=1,2, \ldots, n$.

Necessity. Assume that the Stieltjes polynomial is given by (2.2). Then we have, by virtue of (1.1) and (2.1),

$$
\begin{array}{r}
\int_{a}^{b}\left[\pi_{n+1}(t)-\beta \pi_{n-1}(t)\right]\left[\cdots+c_{1, k}^{n} \pi_{n+1}(t)+c_{0, k}^{n} \pi_{n}(t)+c_{-1, k}^{n} \pi_{n-1}(t)+\cdots\right] d \sigma(t)=0 \\
k=1,2, \ldots, n
\end{array}
$$

which by orthogonality gives

$$
c_{1, k}^{n} \int_{a}^{b} \pi_{n+1}^{2}(t) d \sigma(t)-\beta c_{-1, k}^{n} \int_{a}^{b} \pi_{n-1}^{2}(t) d \sigma(t)=0, \quad k=1,2, \ldots, n,
$$

or equivalently,

$$
c_{1, k}^{n}\left\|\pi_{n+1}\right\|^{2}-\beta c_{-1, k}^{n}\left\|\pi_{n-1}\right\|^{2}=0, \quad k=1,2, \ldots, n .
$$


In view of (2.6), this yields

$$
\left\|\pi_{n}\right\|^{2}\left(\beta c_{1, k}^{n}-c_{-1, k}^{n}\right)=0, \quad k=1,2, \ldots, n,
$$

and since $\left\|\pi_{n}\right\|^{2} \neq 0$, there follows (2.3).

Lemma 2.2. Consider a measure $d \sigma \in \mathcal{M}_{\ell}^{(\alpha, \beta)}[a, b]$ with $\ell=1$. Then in (2.1) there holds

$$
c_{-i, k}^{n}=\beta^{i} c_{i, k}^{n}
$$

for $i=0,1, \ldots, k$, and all $k=0,1, \ldots, n, n \geq 1$.

Proof. We apply induction on $n$. For $n=1$, the induction claim holds trivially when $k=0$, and by means of (1.4) when $k=1$, since

$$
t \pi_{1}(t)=\pi_{2}(t)+\alpha \pi_{1}(t)+\beta \pi_{0}(t)
$$

that is, $c_{1,1}^{1}=1, c_{-1,1}^{1}=\beta$.

Assume now that the claim is true for some index $n$, that is,

$$
\begin{aligned}
t^{k} \pi_{n}(t)= & c_{k, k}^{n} \pi_{n+k}(t)+c_{k-1, k}^{n} \pi_{n+k-1}(t)+\cdots+c_{i, k}^{n} \pi_{n+i}(t)+\cdots+c_{0, k}^{n} \pi_{n}(t) \\
& +\cdots+\beta^{i} c_{i, k}^{n} \pi_{n-i}(t)+\cdots+\beta^{k-1} c_{k-1, k}^{n} \pi_{n-(k-1)}(t) \\
& +\beta^{k} c_{k, k}^{n} \pi_{n-k}(t), \quad k=0,1, \ldots, n
\end{aligned}
$$

we want to prove it for the index $n+1$. The expansion of $t^{k} \pi_{n}(t)$ in terms of the $\pi_{m}$ 's results from applying $k$ times (1.4), solved for the term $t \pi_{n}$. Since (1.4) is assumed to hold with $\ell=1$, we have

$$
t \pi_{m}(t)=\pi_{m+1}(t)+\alpha \pi_{m}(t)+\beta \pi_{m-1}(t)
$$

for all $m \geq 1$. It follows that the coefficients in (2.8) depend only on $\alpha, \beta$ and $k$, and not on $n$. Therefore, replacing $n$ in $\pi_{n}$ by $n+1$ gives the corresponding expansion for $t^{k} \pi_{n+1}(t), k=0,1, \ldots, n$, that is,

$$
\begin{aligned}
& t^{k} \pi_{n+1}(t)= c_{k, k}^{n} \pi_{n+1+k}(t)+c_{k-1, k}^{n} \pi_{n+1+k-1}(t)+\cdots+c_{i, k}^{n} \pi_{n+1+i}(t) \\
&+\cdots+c_{0, k}^{n} \pi_{n+1}(t)+\cdots+\beta^{i} c_{i, k}^{n} \pi_{n+1-i}(t) \\
&+\cdots+\beta^{k-1} c_{k-1, k}^{n} \pi_{n+1-(k-1)}(t)+\beta^{k} c_{k, k}^{n} \pi_{n+1-k}(t) \\
& k=0,1, \ldots, n .
\end{aligned}
$$

This proves the induction claim for the index $n+1$ when $k=0,1, \ldots, n$. It remains to show the claim for $k=n+1$. The expansion for $t^{n+1} \pi_{n+1}(t)$ is obtained by multiplying the expansion for $t^{n} \pi_{n+1}(t)$ by $t$, and then applying (2.9) to each term in the expansion. This yields, in the notation of (2.1),

$$
c_{i, n+1}^{n+1}= \begin{cases}\beta c_{i+1, n}^{n+1}+\alpha c_{i, n}^{n+1}+c_{i-1, n}^{n+1}, & i=1,2, \ldots, n-1, \\ \alpha c_{n, n}^{n+1}+c_{n-1, n}^{n+1}, & i=n, \\ c_{n, n}^{n+1}, & i=n+1\end{cases}
$$


and

$$
c_{-i, n+1}^{n+1}= \begin{cases}\beta c_{-(i-1), n}^{n+1}+\alpha c_{-i, n}^{n+1}+c_{-(i+1), n}^{n+1}, & i=0,1, \ldots, n-1, \\ \beta c_{-(n-1), n}^{n+1}+\alpha c_{-n, n}^{n+1}, & i=n, \\ \beta c_{-n, n}^{n+1}, & i=n+1 .\end{cases}
$$

From (2.10), with $k=n$, there follows

$$
c_{-i, n}^{n+1}=\beta^{i} c_{i, n}^{n+1}, \quad i=0,1, \ldots, n,
$$

which, combined with (2.11) and (2.12), gives

$$
c_{-i, n+1}^{n+1}=\beta^{i} c_{i, n+1}^{n+1}, \quad i=0,1, \ldots, n+1 .
$$

This proves the induction claim for $k=n+1$, and completes the induction.

Theorem 2.3. Consider a measure $d \sigma \in \mathcal{M}_{\ell}^{(\alpha, \beta)}[a, b]$. Then the corresponding Stieltjes polynomials are given by

$$
\pi_{n+1}^{*}(t)=\pi_{n+1}(t)-\beta \pi_{n-1}(t) \text { for } n \geq 2 \ell-1 .
$$

Proof. In order to prove (2.13), it suffices to show, in view of Lemma 2.1, that if (1.4) holds for all $n \geq \ell$, then so does (2.3) for all $n \geq 2 \ell-1$. To this end we apply induction on $\ell$. This requires us to compare the coefficients in the expansion (2.1) in the orthogonal polynomials associated with a $d \sigma_{\ell} \in \mathcal{M}_{\ell}^{(\alpha, \beta)}[a, b]$ with those of the analogous expansion in the (different) orthogonal polynomials associated with a $d \sigma_{\ell+1} \in \mathcal{M}_{\ell+1}^{(\alpha, \beta)}\left[a^{\prime}, b^{\prime}\right]$. This we do by starting from the trivial identity $\pi_{n}(t)=\pi_{n}(t)$ for the polynomial in question and then multiplying both sides repeatedly by $t$, whereby on the right we continuously use (2.9) or the analogous relation from (1.4) (whichever is appropriate) to express the result in terms of higher- and lower-degree polynomials $\pi_{r}$.

The induction claim for $\ell=1$ follows from Lemma 2.2 with $i=1$. Assume now that the claim is true for some index $\ell$; we want to prove it for the index $\ell+1$. Replacing $\ell$ in (1.4) by $\ell+1$ has the effect that the recursion coefficients $\alpha_{\ell}$ and $\beta_{\ell}$ may no longer be equal to $\alpha$ and $\beta$, respectively. As a consequence, the coefficients $c_{ \pm i, k}^{n}$ in (2.1) generated by the above procedure will eventually change as well. In order to prove the induction claim for the index $\ell+1$, we must show that for all $n \geq 2 \ell+1$, the coefficients $c_{ \pm 1, k}^{n}$ that evolve are not affected by the replacement of $\ell$ in (1.4) by $\ell+1$, i.e., $\alpha_{\ell}$ and $\beta_{\ell}$ do not become involved in determining these coefficients. This will be the case for all $k=0,1, \ldots, n-\ell$ since (2.9) still holds for $m \geq \ell+1$. When $k=n-\ell+1$, then $\alpha_{\ell}$ and $\beta_{\ell}$ enter the picture for the first time as parts of the coefficients of $\pi_{\ell}, \pi_{\ell-1}$, and they, as well as lower-order coefficients $\alpha_{\lambda}, \beta_{\lambda}$ with $\lambda<\ell$, continue to be involved for the remaining values of $k=n-\ell+2, n-\ell+3, \ldots, n$. When $k=n$, then $\alpha_{\lambda}, \beta_{\lambda}$ with $\lambda \leq \ell$ are involved in the expansion coefficients of $\pi_{2 \ell-1}, \pi_{2 \ell-2}, \ldots, \pi_{0}$. Since the highest-degree polynomial so affected is $\pi_{2 \ell-1}$, it is clear that when $n \geq 2 \ell+1$, the expansion coefficients associated with $\pi_{n-1}$ and $\pi_{n+1}$, that is, $c_{ \pm 1, k}^{n}, 1 \leq k \leq n$, are independent of $\alpha_{\lambda}, \beta_{\lambda}$ with $\lambda \leq \ell$. This proves the induction claim for the index $\ell+1$, and completes the induction.

The following proposition will be useful in the development of $\S 3$. 
Proposition 2.4. Consider a measure $d \sigma \in \mathcal{M}_{\ell}^{(\alpha, \beta)}[a, b]$ and let $\tau_{\nu}$ be the zeros of the corresponding orthogonal polynomial $\pi_{n}$. Then

$$
\pi_{n+1}\left(\tau_{\nu}\right)=\frac{1}{2} \pi_{n+1}^{*}\left(\tau_{\nu}\right), \quad \nu=1,2, \ldots, n,
$$

for all $n \geq 2 \ell-1$.

Proof. Let $n \geq 2 \ell-1$. First, (2.13) gives

$$
\pi_{n+1}^{*}\left(\tau_{\nu}\right)=\pi_{n+1}\left(\tau_{\nu}\right)-\beta \pi_{n-1}\left(\tau_{\nu}\right) .
$$

Since $\tau_{\nu}$ is a zero of $\pi_{n}$, we have by (1.4) that

$$
\beta \pi_{n-1}\left(\tau_{\nu}\right)=-\pi_{n+1}\left(\tau_{\nu}\right)
$$

which, inserted into (2.15), yields (2.14).

\section{Gauss-Kronrod quadrature formulae}

The Gauss-Kronrod formula (1.2) is said to have the interlacing property if the nodes $\tau_{\nu}, \tau_{\mu}^{*}$ are real and satisfy, when ordered decreasingly,

$$
\tau_{n+1}^{*}<\tau_{n}<\tau_{n}^{*}<\cdots<\tau_{2}^{*}<\tau_{1}<\tau_{1}^{*} .
$$

Formula (1.2) is said to have the inclusion property if all nodes $\tau_{\nu}, \tau_{\mu}^{*}$ are contained in the closed interval $[a, b]$. Clearly, if (3.1) holds, the inclusion property is equivalent to

$$
a \leq \tau_{n+1}^{*} \text { and } \tau_{1}^{*} \leq b .
$$

If $d \sigma \in \mathcal{M}_{\ell}^{(\alpha, \beta)}[a, b]$, then trivially $\alpha_{n} \rightarrow \alpha, \beta_{n} \rightarrow \beta$ as $n \rightarrow \infty$, and it follows $[3$, p. 121] that

$$
[\alpha-2 \sqrt{\beta}, \alpha+2 \sqrt{\beta}]
$$

is the "limiting spectral interval" of $d \sigma$. It may well be, however, that $d \sigma$ has support points outside the interval (3.3) (cf. [3, Exercise 4.6, p. 128]), but for inclusion results we will assume the following property.

Property A. The measure $d \sigma \in \mathcal{M}_{\ell}^{(\alpha, \beta)}[a, b]$ is such that

$$
a=\alpha-2 \sqrt{\beta}, \quad b=\alpha+2 \sqrt{\beta} .
$$

Before we state and prove the properties of the quadrature formula (1.2) announced in $\S 1$, we add another lemma in the spirit of Lemma 2.2 and Theorem 2.3.

Lemma 3.1. Consider a measure $d \sigma \in \mathcal{M}_{\ell}^{(\alpha, \beta)}[a, b]$. Then in (2.1) there holds, for all $n \geq 2 \ell-1$,

$$
c_{-i, n}^{n}=\beta^{i} c_{i, n}^{n}, i=0,1, \ldots, n-2 \ell+2 .
$$

Proof. For $\ell=1$, this is Lemma 2.2 with $k=n$. The proof for general $\ell$ is again by induction, very much along the lines of the proof of Theorem 2.3. The details are left to the reader.

Theorem 3.2. Consider a measure $d \sigma \in \mathcal{M}_{\ell}^{(\alpha, \beta)}[a, b]$. Then the following holds :

(a) The Gauss-Kronrod formula (1.2) has the interlacing property for all $n \geq$ $2 \ell-1$.

(b) If $d \sigma$ has Property A, then the inclusion property holds for all $n \geq 2 \ell-1$.

(c) All weights $\sigma_{\nu}, \sigma_{\mu}^{*}$ in (1.2) are positive for each $n \geq 2 \ell-1$.

(d) The formula (1.2) has degree of exactness (at least) $4 n-2 \ell+2$ if $n \geq 2 \ell-1$. 
Remark. In part (b) of this theorem, Property A can be replaced by assuming the two inequalities in (3.8).

Proof. (a) Let $n \geq 2 \ell-1$. Proposition 2.4, in view of the separation property for the zeros of $\pi_{n}$ and $\pi_{n+1}$ (see [13, Theorem 3.3.2]), implies

$$
\operatorname{sign} \pi_{n+1}^{*}\left(\tau_{\nu}\right)=\operatorname{sign} \pi_{n+1}\left(\tau_{\nu}\right)=(-1)^{\nu}, \quad \nu=1,2, \ldots, n .
$$

In addition, it is clear that

$$
\begin{gathered}
\lim _{t \rightarrow \infty} \pi_{n+1}^{*}(t)=\infty, \\
\lim _{t \rightarrow-\infty} \pi_{n+1}^{*}(t)=(-1)^{n+1} \infty .
\end{gathered}
$$

From (3.6) and (3.7) there follows that the $\tau_{\mu}^{*}$ are real and satisfy (3.1). This proves the interlacing property.

(b) Let $n \geq 2 \ell-1$. Since (3.1) is true, the inclusion property comes down to showing that (3.2) holds. A necessary and sufficient condition for that is

$$
(-1)^{n+1} \pi_{n+1}^{*}(a) \geq 0 \text { and } \pi_{n+1}^{*}(b) \geq 0,
$$

which, on account of (2.13), is equivalent to

$$
\beta \leq \frac{\pi_{n+1}(a)}{\pi_{n-1}(a)} \text { and } \beta \leq \frac{\pi_{n+1}(b)}{\pi_{n-1}(b)} .
$$

Assuming Property A, we now prove both these inequalities. Beginning with the second, we set $t=b$ in (1.4), to get, using the second relation in (3.4),

$$
\pi_{n+1}(b)=2 \sqrt{\beta} \pi_{n}(b)-\beta \pi_{n-1}(b), \quad n \geq \ell .
$$

Dividing both sides of (3.9) by $\pi_{n}(b)$, and letting $q_{n}=\pi_{n}(b) / \pi_{n-1}(b)$, we obtain

$$
q_{n+1}=2 \sqrt{\beta}-\frac{\beta}{q_{n}}, \quad n \geq \ell .
$$

Subtracting $q_{n}$ from both sides gives

$$
q_{n+1}-q_{n}=-\frac{\left(q_{n}-\sqrt{\beta}\right)^{2}}{q_{n}}, \quad n \geq \ell .
$$

Since $q_{n}>0$ for $n \geq 1$, there follows from (3.10) that $q_{n}$ is a decreasing sequence for $n \geq \ell$ and hence converges to, say, $q$ as $n \rightarrow \infty$. Thus, $q_{n} \geq q$ for $n \geq \ell$. Multiplying both sides of (3.10) by $q_{n}$, and then taking the limit as $n \rightarrow \infty$, we immediately obtain $q=\sqrt{\beta}$, hence

$$
q_{n} \geq \sqrt{\beta}, \quad n \geq \ell .
$$

Now,

$$
\frac{\pi_{n+1}(b)}{\pi_{n-1}(b)}=\frac{\pi_{n+1}(b)}{\pi_{n}(b)} \cdot \frac{\pi_{n}(b)}{\pi_{n-1}(b)}=q_{n+1} q_{n},
$$

which by (3.11) yields the second inequality in (3.8).

For the first inequality, the proof is analogous. One now defines $q_{n}$ by $q_{n}=$ $\pi_{n}(a) / \pi_{n-1}(a)$ and shows that $q_{n}$ for $n \geq \ell$ is a (negative) increasing sequence converging to $-\sqrt{\beta}$, hence,

$$
q_{n} \leq-\sqrt{\beta}, \quad n \geq \ell,
$$

from which the first inequality in (3.8) follows as before. 
(c) The weights $\sigma_{\nu}$ are given by the formula

$$
\sigma_{\nu}=\lambda_{\nu}+\frac{\left\|\pi_{n}\right\|^{2}}{\pi_{n}^{\prime}\left(\tau_{\nu}\right) \pi_{n+1}^{*}\left(\tau_{\nu}\right)}, \quad \nu=1,2, \ldots, n
$$

(see [9, Theorem 2]), where $\lambda_{\nu}=\lambda_{\nu}^{(n)}$ are the weights of the $n$-point Gauss formula relative to $d \sigma$, known to be all positive, and $\|\cdot\|$ denotes the $L_{2}$-norm. Also, the $\lambda_{\nu}$ can be represented by

$$
\lambda_{\nu}=-\frac{\left\|\pi_{n}\right\|^{2}}{\pi_{n}^{\prime}\left(\tau_{\nu}\right) \pi_{n+1}\left(\tau_{\nu}\right)}, \quad \nu=1,2, \ldots, n
$$

(see [13, Eq. (3.4.7)]). Let $n \geq 2 \ell-1$. Then (3.12), by virtue of (2.14) and (3.13), yields

$$
\sigma_{\nu}=\frac{1}{2} \lambda_{\nu}, \quad \nu=1,2, \ldots, n,
$$

from which the positivity of the $\sigma_{\nu}$ follows immediately.

The positivity of the $\sigma_{\mu}^{*}$ is equivalent to the interlacing property (see [9, Theorem 1]) already proved in (a).

(d) Let $n \geq 2 \ell-1$. To prove that the degree of exactness is (at least) $4 n-2 \ell+2$, it suffices to show

$$
\int_{a}^{b} \pi_{n+1}^{*}(t) t^{k} \pi_{n}(t) d \sigma(t)=0, \quad k=0,1, \ldots, 2 n-2 \ell+1
$$

(see [5, Corollary]). By (1.1), this is true for $k=0,1, \ldots, n$. For the remaining values $k=n+1, n+2, \ldots, 2 n-2 \ell+1$ we can write (3.15) in view of (2.13) as

$$
\int_{a}^{b} t^{k}\left[\pi_{n+1}(t)-\beta \pi_{n-1}(t)\right] t^{n} \pi_{n}(t) d \sigma(t)=0, \quad k=0,1, \ldots, n-2 \ell+1 .
$$

By Lemma 3.1, we can write

$$
\begin{aligned}
& t^{n} \pi_{n}(t)= c_{n, n}^{n} \pi_{2 n}(t)+\cdots+c_{n-2 \ell+2, n}^{n} \pi_{2 n-2 \ell+2}(t)+\cdots+c_{i, n}^{n} \pi_{n+i}(t) \\
&+\cdots+c_{0, n}^{n} \pi_{n}(t)+\cdots+\beta^{i} c_{i, n}^{n} \pi_{n-i}(t) \\
&+\cdots+\beta^{n-2 \ell+2} c_{n-2 \ell+2, n}^{n} \pi_{2 \ell-2}(t)+\cdots+c_{-n, n}^{n} \pi_{0}(t) \\
& n \geq 2 \ell-1 .
\end{aligned}
$$

Similarly, in the expansion

$$
t^{k}\left[\pi_{n+1}(t)-\beta \pi_{n-1}(t)\right]=\sum_{i=-(k+1)}^{k+1} d_{i, k+1}^{n} \pi_{n+i}
$$

we have $d_{0, k+1}^{n}=0$, and $d_{-i, k+1}^{n}=-\beta^{i} d_{i, k+1}^{n}$ for each $i=1,2, \ldots, k+1$, and for all $k=0,1, \ldots, n-2 \ell+1, n \geq 2 \ell-1$. The proof goes by induction on $\ell$. Let 
$\ell=1$. First, we have from Lemma 2.2

$$
\begin{aligned}
t^{k} \pi_{n-1}(t)= & c_{k, k}^{n-1} \pi_{n-1+k}(t)+c_{k-1, k}^{n-1} \pi_{n-1+k-1}(t)+\cdots+c_{i+1, k}^{n-1} \pi_{n-1+i+1}(t) \\
& +c_{i, k}^{n-1} \pi_{n-1+i}(t)+c_{i-1, k}^{n-1} \pi_{n-1+i-1}(t)+\cdots+c_{2, k}^{n-1} \pi_{n+1}(t) \\
& +c_{1, k}^{n-1} \pi_{n}(t)+c_{0, k}^{n-1} \pi_{n-1}(t)+\beta c_{1, k}^{n-1} \pi_{n-2}(t)+\beta^{2} c_{2, k}^{n-1} \pi_{n-3}(t) \\
& +\cdots+\beta^{i-1} c_{i-1, k}^{n-1} \pi_{n-1-(i-1)}(t)+\beta^{i} c_{i, k}^{n-1} \pi_{n-1-i}(t) \\
& +\beta^{i+1} c_{i+1, k}^{n-1} \pi_{n-1-(i+1)}(t)+\cdots+\beta^{k-1} c_{k-1, k}^{n-1} \pi_{n-1-(k-1)}(t) \\
& +\beta^{k} c_{k, k}^{n-1} \pi_{n-1-k}(t), \quad k=0,1, \ldots, n-1, \quad n \geq 1 .
\end{aligned}
$$

Since (1.4), with $\ell=1$, holds for all $n \geq 1$, the coefficients in this expansion are given in terms of $\alpha, \beta$ and $k$ only. Therefore, replacing $n-1$ in $\pi_{n-1}$ by $n+1$ gives the corresponding expansion for $t^{k} \pi_{n+1}(t), k=0,1, \ldots, n-1$, that is,

$$
\begin{aligned}
t^{k} \pi_{n+1}(t)= & c_{k, k}^{n-1} \pi_{n+1+k}(t)+c_{k-1, k}^{n-1} \pi_{n+1+k-1}(t)+\cdots+c_{i+1, k}^{n-1} \pi_{n+1+i+1}(t) \\
& +c_{i, k}^{n-1} \pi_{n+1+i}(t)+c_{i-1, k}^{n-1} \pi_{n+1+i-1}(t)+\cdots+c_{2, k}^{n-1} \pi_{n+3}(t) \\
& +c_{1, k}^{n-1} \pi_{n+2}(t)+c_{0, k}^{n-1} \pi_{n+1}(t)+\beta c_{1, k}^{n-1} \pi_{n}(t)+\beta^{2} c_{2, k}^{n-1} \pi_{n-1}(t) \\
& +\cdots+\beta^{i-1} c_{i-1, k}^{n-1} \pi_{n+1-(i-1)}(t)+\beta^{i} c_{i, k}^{n-1} \pi_{n+1-i}(t) \\
& +\beta^{i+1} c_{i+1, k}^{n-1} \pi_{n+1-(i+1)}(t)+\cdots+\beta^{k-1} c_{k-1, k}^{n-1} \pi_{n+1-(k-1)}(t) \\
& +\beta^{k} c_{k, k}^{n-1} \pi_{n+1-k}(t), \quad k=0,1, \ldots, n-1, \quad n \geq 1
\end{aligned}
$$

(see also the proof of Lemma 2.2). Adding (3.18) multiplied by $-\beta$ to (3.19), we get

$$
\begin{aligned}
& t^{k}\left[\pi_{n+1}(t)-\beta \pi_{n-1}(t)\right]=c_{k, k}^{n-1} \pi_{n+k+1}(t)+c_{k-1, k}^{n-1} \pi_{n+k}(t) \\
& \quad+\cdots+\left(c_{i-1, k}^{n-1}-\beta c_{i+1, k}^{n-1}\right) \pi_{n+i}(t)+\cdots+\left(c_{0, k}^{n-1}-\beta c_{2, k}^{n-1}\right) \pi_{n+1}(t) \\
& \quad+\left(\beta c_{1, k}^{n-1}-\beta c_{1, k}^{n-1}\right) \pi_{n}(t)+\left(\beta^{2} c_{2, k}^{n-1}-\beta c_{0, k}^{n-1}\right) \pi_{n-1}(t) \\
& \quad+\cdots+\left(\beta^{i+1} c_{i+1, k}^{n-1}-\beta^{i} c_{i-1, k}^{n-1}\right) \pi_{n-i}(t)+\cdots-\beta^{k} c_{k-1, k}^{n-1} \pi_{n-k}(t) \\
& \quad-\beta^{k+1} c_{k, k}^{n-1} \pi_{n-(k+1)}(t),
\end{aligned}
$$

or equivalently,

$$
\begin{gathered}
t^{k}\left[\pi_{n+1}(t)-\beta \pi_{n-1}(t)\right]=c_{k, k}^{n-1} \pi_{n+k+1}(t)+c_{k-1, k}^{n-1} \pi_{n+k}(t) \\
+\cdots+\left(c_{i-1, k}^{n-1}-\beta c_{i+1, k}^{n-1}\right) \pi_{n+i}(t)+\cdots+\left(c_{0, k}^{n-1}-\beta c_{2, k}^{n-1}\right) \pi_{n+1}(t) \\
-\beta\left(c_{0, k}^{n-1}-\beta c_{2, k}^{n-1}\right) \pi_{n-1}(t)-\beta^{i}\left(c_{i-1, k}^{n-1}-\beta c_{i+1, k}^{n-1}\right) \pi_{n-i}(t) \\
-\cdots-\beta^{k} c_{k-1, k}^{n-1} \pi_{n-k}(t)-\beta^{k+1} c_{k, k}^{n-1} \pi_{n-(k+1)}(t), \\
k=0,1, \ldots, n-1, \quad n \geq 1,
\end{gathered}
$$

which proves the induction claim for $\ell=1$. 
Assume that the claim is true for some index $\ell$, that is,

$$
\begin{gathered}
t^{k}\left[\pi_{n+1}(t)-\beta \pi_{n-1}(t)\right]=d_{k+1, k+1}^{n} \pi_{n+k+1}(t)+\cdots+d_{i, k+1}^{n} \pi_{n+i}(t) \\
+\cdots+d_{1, k+1}^{n} \pi_{n+1}(t)-\beta d_{1, k+1}^{n} \pi_{n-1}(t)-\cdots-\beta^{i} d_{i, k+1}^{n} \pi_{n-i}(t) \\
-\cdots-\beta^{k+1} d_{k+1, k+1}^{n} \pi_{n-(k+1)}(t) \\
\quad k=0,1, \ldots, n-2 \ell+1, n \geq 2 \ell-1
\end{gathered}
$$

we want to prove it for the index $\ell+1$. Replacing $\ell$ in (1.4) by $\ell+1$ has the effect of making the recursion coefficients $\alpha_{\ell}$ and $\beta_{\ell}$ in general different from $\alpha$ and $\beta$, respectively. As a consequence, the coefficients in the expansion of $t^{k}\left[\pi_{n+1}(t)-\beta \pi_{n-1}(t)\right]$ change as well, and $\alpha_{\ell}$ and $\beta_{\ell}$ enter these coefficients as $k$ advances. Indeed, for $k=0,1, \ldots, n-\ell-1$, the coefficients are the same as before the replacement. When $k=n-\ell$, then $\alpha_{\ell}$ and $\beta_{\ell}$ enter the scene for the first time as parts of the coefficients of $\pi_{\ell}, \pi_{\ell-1}$ (see also the proof of Theorem 2.3). However, $n-\ell>n-2 \ell+1$ for $\ell>1$. Hence, the expansion coefficients in (3.20) are independent of $\alpha_{\ell}$ and $\beta_{\ell}$, and therefore the coefficients in the expansion of $t^{k}\left[\pi_{n+1}(t)-\beta \pi_{n-1}(t)\right], k=0,1, \ldots, n-2 \ell-1, n \geq 2 \ell+1$, are the same as before the replacement of $\ell$ in (1.4) by $\ell+1$. This proves the induction claim for index $\ell+1$, and completes the induction.

Now, (3.16) can be verified by multiplying together the expansions (3.17) and (3.20), and by using orthogonality and the fact that

$$
\left\|\pi_{n+i}\right\|^{2}-\beta^{2 i}\left\|\pi_{n-i}\right\|^{2}=0, \quad i=0,1, \ldots, n-\ell+1, \quad n \geq \ell-1
$$

(cf. [4, Eq. (5.3)]).

\section{INTERPOLATORY QUADRATURE FORMULAE}

In this section we show that, under the assumption $d \sigma \in \mathcal{M}_{\ell}^{(\alpha, \beta)}[a, b]$, formula (1.3) has real nodes, all included in the closed interval $[a, b]$ (if $d \sigma$ has Property A), and positive weights for all $n \geq 2 \ell-1$. In addition, we determine the precise degree of exactness of (1.3).

Theorem 4.1. Consider a measure $d \sigma \in \mathcal{M}_{\ell}^{(\alpha, \beta)}[a, b]$. Then the following holds :

(a) The interpolatory formula (1.3) has real nodes which, if $d \sigma$ has Property A, are all contained in the closed interval $[a, b]$, for each $n \geq 2 \ell-1$.

(b) All weights $w_{\mu}^{*}$ in (1.3) are positive for each $n \geq 2 \ell-1$.

(c) The precise degree of exactness of (1.3) is $2 n-1$ if $n \geq 2 \ell-1$.

Proof. (a) The assertions follow from Theorem 3.2 (a), (b).

(b) Setting $f(t)=\pi_{n+1}^{*}(t) /\left(t-\tau_{\mu}^{*}\right)$ in the interpolatory formula (1.3), we get

$$
w_{\mu}^{*}=\frac{1}{\pi_{n+1}^{* \prime}\left(\tau_{\mu}^{*}\right)} \int_{a}^{b} \frac{\pi_{n+1}^{*}(t)}{t-\tau_{\mu}^{*}} d \sigma(t), \mu=1,2, \ldots, n+1 .
$$

That same substitution in the Gauss-Kronrod formula (1.2) and in the $n$-point Gauss formula relative to $d \sigma$,

$$
\int_{a}^{b} f(t) d \sigma(t)=\sum_{\nu=1}^{n} \lambda_{\nu} f\left(\tau_{\nu}\right)+R_{n}^{G}(f),
$$


where $\tau_{\nu}=\tau_{\nu}^{(n)}$ are the zeros of $\pi_{n}$ and $\lambda_{\nu}=\lambda_{\nu}^{(n)}$ the Christoffel numbers, gives

$$
\int_{a}^{b} \frac{\pi_{n+1}^{*}(t)}{t-\tau_{\mu}^{*}} d \sigma(t)=\sum_{\nu=1}^{n} \sigma_{\nu} \frac{\pi_{n+1}^{*}\left(\tau_{\nu}\right)}{\tau_{\nu}-\tau_{\mu}^{*}}+\sigma_{\mu}^{*} \pi_{n+1}^{* \prime}\left(\tau_{\mu}^{*}\right)
$$

and

$$
\int_{a}^{b} \frac{\pi_{n+1}^{*}(t)}{t-\tau_{\mu}^{*}} d \sigma(t)=\sum_{\nu=1}^{n} \lambda_{\nu} \frac{\pi_{n+1}^{*}\left(\tau_{\nu}\right)}{\tau_{\nu}-\tau_{\mu}^{*}}
$$

respectively. Let $n \geq 2 \ell-1$. Equating the right sides of (4.2) and (4.3), we find, in view of (3.14),

$$
\sum_{\nu=1}^{n} \sigma_{\nu} \frac{\pi_{n+1}^{*}\left(\tau_{\nu}\right)}{\tau_{\nu}-\tau_{\mu}^{*}}=\sigma_{\mu}^{*} \pi_{n+1}^{* \prime}\left(\tau_{\mu}^{*}\right)
$$

which, inserted into (4.2), yields

$$
\int_{a}^{b} \frac{\pi_{n+1}^{*}(t)}{t-\tau_{\mu}^{*}} d \sigma(t)=2 \sigma_{\mu}^{*} \pi_{n+1}^{* \prime}\left(\tau_{\mu}^{*}\right)
$$

Now, (4.1), by virtue of (4.4), implies

$$
w_{\mu}^{*}=2 \sigma_{\mu}^{*}, \quad \mu=1,2, \ldots, n+1 .
$$

By Theorem $3.2(\mathrm{c})$, the positivity of $w_{\mu}^{*}$ follows.

(c) The precise degree of exactness of (1.3) is $n+k$, where $k$ is the unique integer satisfying

$$
\int_{a}^{b} \pi_{n+1}^{*}(t) p(t) d \sigma(t) \begin{cases}=0 & \text { for all } p \in \mathbb{P}_{k-1} \\ \neq 0 & \text { for some } p \in \mathbb{P}_{k}\end{cases}
$$

(see $[4, \S 1.3]$ ). Now, for $n \geq 2 \ell-1$, we have by orthogonality, in view of (2.13),

$$
\begin{gathered}
\int_{a}^{b} \pi_{n+1}^{*}(t) p(t) d \sigma(t) \\
=\int_{a}^{b}\left[\pi_{n+1}(t)-\beta \pi_{n-1}(t)\right] p(t) d \sigma(t) \begin{cases}=0 & \text { for all } p \in \mathbb{P}_{n-2}, \\
=-\left\|\pi_{n}\right\|^{2} \neq 0 & \text { for } p=\pi_{n-1} .\end{cases}
\end{gathered}
$$

Thus, the precise degree of exactness of (1.3) is $2 n-1$.

\section{ACKNOWLEDGMENT}

The authors are indebted to Professor T.S. Chihara for pointing them to the references [1] and [8].

\section{REFERENCES}

1. Wm. R. Allaway, "The identification of a class of orthogonal polynomial sets", Ph.D. Thesis, Univ. Alberta, 1972.

2. F. Caliò, W. Gautschi and E. Marchetti, "On computing Gauss-Kronrod quadrature formulae", Math. Comp., v. 47, 1986, pp. 639-650. MR 88a:65028

3. T. S. Chihara, An Introduction to Orthogonal Polynomials, Gordon and Breach, New York, 1978. MR 58:1979

4. W. Gautschi, "A survey of Gauss-Christoffel quadrature formulae", in E. B. Christoffel (P. L. Butzer and F. Fehér, eds.), Birkhäuser, Basel, 1981, pp. 72-147. MR 83g:41031

5. W. Gautschi, "Gauss-Kronrod quadrature - a survey", in Numerical Methods and Approximation Theory III (G. V. Milovanović, ed.), Faculty of Electronic Engineering, Univ. Niš, Niš, 1988, pp. 39-66. MR 89k:41035 
6. W. Gautschi and S. E. Notaris, "Gauss-Kronrod quadrature formulae for weight functions of Bernstein-Szegö type", J. Comput. Appl. Math., v. 25, 1989, pp. 199-224; erratum in: J. Comput. Appl. Math., v. 27, 1989, p. 429. MR 90d:65045; 90m:65055

7. W. Gautschi and T. J. Rivlin, "A family of Gauss-Kronrod quadrature formulae", Math. Comp., v. 51, 1988, pp. 749-754. MR 89m:65029

8. A. Máté, P. Nevai and W. Van Assche, "The supports of measures associated with orthogonal polynomials and the spectra of the related self-adjoint operators", Rocky Mountain J. Math., v. 21, 1991, pp. 501-527. MR 92i:42015

9. G. Monegato, "A note on extended Gaussian quadrature rules", Math. Comp., v. 30, 1976, pp. 812-817. MR 55:13746

10. G. Monegato, "Stieltjes polynomials and related quadrature rules", SIAM Rev., v. 24, 1982, pp. 137-158. MR 83d:65067

11. S. E. Notaris, "Gauss-Kronrod quadrature formulae for weight functions of Bernstein-Szegö type, II", J. Comput. Appl. Math., v. 29, 1990, pp. 161-169. MR 91b:65030

12. F. Peherstorfer, "Weight functions admitting repeated positive Kronrod quadrature", BIT, v. 30, 1990, pp. 145-151. MR 91e:65043

13. G. Szegö, Orthogonal Polynomials, Colloquium Publications, v. 23, 4th ed., American Mathematical Society, Providence, R.I., 1975. MR 51:8724

Department of Computer Sciences, Purdue University, West Lafayette, Indiana 47907-1398

E-mail address: wxg@cs.purdue.edu

Department of Communications and Mass Media, National and Capodistrian UniverSity of Athens, GR-10562, Athens, Greece 